\title{
AN INTRODUCTION TO THE THEORY OF IDEALS IN LINEAR ASSOCIATIVE ALGEBRAS*
}

\author{
BY \\ C. C. MACDUFFEE
}

1. Introduction. With the development of the number theory of linear algebras, it was natural that attempts should be made to extend to these domains of integrity the theory of ideal numbers. But it is evident from the few domains which have been examined that one cannot expect an extension of this theory in its entirety. For instance, Hurwitz $\dagger$ has investigated the number theory of quaternions by using right and left ideals, and has found that they are powerless to introduce unique factorization into this algebra. Moreover, Speiserł has recently investigated the properties of right, left and two-sided ideals in semi-simple algebras, remarking in the introduction to his paper that some of the most remarkable properties of ideals are "but foreign adjuncts which are essentially restricted to algebraic number fields."

Although it is historically true that ideals were introduced into algebraic number theory to establish unique factorization, it should be observed that this is a secondary function of ideals. Primarily they establish the property that every two numbers have a greatest common divisor expressible linearly in terms of the numbers. In algebraic fields this property implies unique factorization but in the general linear algebra it does not-hence the success of the ideal theory in algebraic fields and its partial failure in the more general domain.

The method which is here used in developing the theory of ideals is different from the usual one. It depends upon a correspondence $\S$ between ideals and matrices whose elements are rational integers, and the only kind of multiplication which is employed is ordinary matric multiplication. Ideal multiplication, which plays such an important rôle in the usual treatment of ideals in algebraic fields, but which causes so much difficulty in non-commutative domains, is not employed in this paper.

\footnotetext{
* Presented to the Society, September 9, 1926; received by the editors in September, 1927.

$\dagger$ A. Hurwitz, Vorlesungen über die Zahlentheorie der Quaternionen, Berlin, Springer, 1919. pp. 8-49.

$\ddagger$ A. Speiser, Vierteljahrsschrift der Naturforschenden Gesellschaft in Zürich, vol. 71 (1926),

$\S$ Applied to quadratic ideals in a recent paper by the author, Annals of Mathematics, (2), vol. 29 (1927-28), pp. 199-214.
} 
The fundamental theorem in the present development (Theorem 4) serves as a substitute for the commutative law in the multiplication of these ideal matrices. From this starting point we proceed to establish for general algebras many of the properties which ideals in algebraic fields are known to possess, including the important concept of class number. Furthermore, we obtain some properties of ideals in all semi-simple algebras which have not previously been found even in the special case of algebraic fields.

The writer is indebted to his colleague Professor Blumberg for helpful suggestions.

2. Definition of ideal. We suppose that $\mathfrak{A}$ is a rational semi-simple algebra of order $n$ with a principal unit, and that $\subseteq$ is a set of integral elements of order $n$ in $\mathfrak{A}$ according to the definition of Dickson.* We suppose that the basal numbers $e_{1}, e_{2}, \cdots, e_{n}$ of $\mathfrak{A}$ form a basis for $\mathfrak{S}, e_{1}$ being the principal unit. $\dagger$ We shall define a left (right) ideal $\Omega$ to be a set of numbers of $\subseteq$ which is closed under addition and subtraction and under multiplication on the left (right) by the numbers of $\subseteq . \ddagger$

We shall develop only the theory of left ideals, as the theory of right ideals is obviously parallel.

In the usual manner§ we can show that every left ideal $\Omega$ has a basis composed of $r \leqq n$ linearly independent numbers $\omega_{1}, \omega_{2}, \cdots, \omega_{r}$. That is, the numbers of $\Omega$ are represented by the form

$$
k_{1} \omega_{1}+k_{2} \omega_{2}+\cdots+k_{r} \omega_{r}
$$

where $k_{1}, k_{2}, \cdots, k_{r}$ are rational integral variables. In particular the $\omega_{i}$ may be chosen in the form

$$
\begin{aligned}
\omega_{1}= & a_{11} e_{1}, \\
\omega_{2}= & a_{21} e_{1}+a_{22} e_{2}, \\
\cdot & \cdot \cdot \cdot \cdot \cdot \cdot \cdot \\
\omega_{r}= & a_{r 1} e_{1}+a_{r 2} e_{2}+\cdots+a_{r r} e_{r}
\end{aligned}
$$

where each $a_{i i}>0$.

* L. E. Dickson, Algebras and their Arithmetics, University of Chicago Press, 1923, p. 141. Algebren und ihre Zahlentheorie, Zürich, 1927, p. 155. These books will be referred to as Dickson I and Dickson II, respectively.

† Dickson I, p. 163, II, p. 212.

$\ddagger$ This is more general than the definition of Speiser, which further specifies that the set $\Re$ be of order $n$, thus excluding what we shall call singular ideals. See Dickson II, p. 270.

8 Dickson II, p. 270. Landau, Theorie der algebraischen Zahlen und der Ideale, Leipzig, 1918, p. 29, Theorem 99 . 
This number $r$, which is evidently the number of linearly independent numbers in every basis of $\Omega$, is called the rank of the ideal. The ideal is called singular or non-singular according as $r<n$ or $r=n$.

3. Some properties of ideals. Every number $a$ satisfies its rank equation*

$$
\omega^{r}+c_{1} \omega^{r-1}+\cdots+c_{r-1} \omega+N(a)=0 .
$$

From the definition of ideal it follows that if $a$ is in $\Re$, then

$$
N(a)=\left(-a^{r-1}-c_{1} a^{r-2}-\cdots-c_{r-1}\right) a
$$

is also in $\Omega$. Hence $\Re$ contains rational integers unless every number of $\Omega$ is of norm 0 .

Let $p$ be the smallest positive integer in the ideal $\Omega$. Let $c$ be any rational integer in $\Re$. Then we may write $c=k p+r$ where $0 \leqq r<p$. Since $c$ and $p$ are in $\Omega$, so is $c-k p=r$. But $p$ was minimal, so $r=0$ and $c=k p$. Hence if not every number of $\Omega$ is of norm $0, \Omega$ contains infinitely many rational integers, each an integral multiple of a smallest positive integer. If $a$ is a number of $\Omega$, so is $N(a)$.

Let $\Omega$ be an ideal containing a positive integer $p$. Since $\subseteq$ is of order $n$, it contains $n$ linearly independent numbers $s_{1}, s_{2}, \cdots, s_{n}$. Then $\Re$ contains the linearly independent numbers $s_{1} p, s_{2} p, \cdots, s_{n} p$. It follows that if $\Omega$ contains a positive integer, the numbers $\omega_{1}, \omega_{2}, \cdots, \omega_{n}$ of every basis are linearly independent and $\Omega$ is of rank $n$.

Let $\omega_{1}^{\prime}, \omega_{2}^{\prime}, \cdots, \omega_{n}^{\prime}$ constitute a second basis for an ideal $\Omega$ of rank $n$. There are rational integers $a_{i j}$ and $b_{i j}$ such that

$$
\omega_{i}=\sum_{k} a_{i k} \omega_{k}^{\prime}, \quad \omega_{i}^{\prime}=\sum_{k} b_{i k} \omega_{k} \quad(i=1,2, \cdots, n) .
$$

Then

$$
\omega_{i}=\sum_{j, k} a_{i j} b_{j k} \omega_{k},
$$

and since the basal numbers are linearly independent,

$$
\sum_{j} a_{i j} b_{j k}=\delta_{i k} \quad(i, k=1,2, \cdots, n)
$$

so that $\left(a_{r s}\right)$ is an integral matrix of determinant \pm 1 .

Conversely, if $\left(a_{r s}\right)$ is an integral matrix of determinant \pm 1 , its inverse is likewise an integral matrix of determinant \pm 1 and will serve as the matrix $\left(b_{r s}\right)$ in (3.1). If the $\omega_{i}^{\prime}$ constitute a basis for $\Re$, so do the $\omega_{i}$ as determined by

* Dickson I, p. 113. 
(3.1). Thus if $\Omega$ is an ideal having a basis $\omega_{1}, \omega_{2}, \cdots, \omega_{n}$ composed of linearly independent numbers, every basis of $\Re$ is given by

$$
\omega_{i}^{\prime}=\sum_{j} a_{i j} \omega_{j} \quad(i=1,2, \cdots, n)
$$

where $\left(a_{r s}\right)$ is an integral matrix of determinant \pm 1 .

If $\Omega$ has a basis $\omega_{1}, \omega_{2}, \cdots, \omega_{n}$, we may write

$$
\omega_{i}=\sum_{j} g_{i j} e_{j} \quad(i=1,2, \cdots, n),
$$

where the $g_{i j}$ are rational integers, in terms of the basal numbers $e_{1}, e_{2}$, $\cdots, e_{n}$ of $\subseteq$. We define the norm $N(\Omega)$ of the ideal $\Omega$ to be the absolute value of the determinant $\left|g_{r s}\right|$. If $N(\Omega)=0$, the $\omega_{i}$ are linearly dependent and the rank $r$ of $\Omega$ is less than $n$; i.e., $\Omega$ is singular.

Suppose that $\omega_{1}, \omega_{2}, \cdots, \omega_{n}$ constitute a linearly independent basis for 凡. If $\omega_{1}^{\prime}, \omega_{2}^{\prime}, \cdots, \omega_{n}^{\prime}$ is any basis for $\Re$, we have

$$
\omega_{i}^{\prime}=\sum_{j} a_{i j} \omega_{j}=\sum_{j, k} a_{i j} g_{j k} e_{k} \equiv \sum_{k} g_{i k}^{\prime} e_{k} \quad(i=1,2, \cdots, n)
$$

where $\left|a_{r s}\right|= \pm 1$. Hence

which implies that

$$
\left(a_{r s}\right)\left(g_{r s}\right)=\left(g_{r s}^{\prime}\right)
$$

$$
\text { absolute value }\left|g_{r_{\bullet}}\right|=\text { absolute value }\left|g_{r e}\right| \neq 0,
$$

so that the value of $N(\Omega)$ is independent of the basis chosen. In other words, $N(\Omega)$ is an invariant under change of basis and is therefore a positive integer intrinsically connected with the ideal $\Omega$.

Let us now suppose that $\Omega$ is an ideal for which $N(\Omega) \neq 0$. From (3.2) we have

$$
N(\Re) e_{i}=\sum_{i} d_{i j} \omega_{j} \quad(i=1,2, \cdots, n)
$$

where the $d_{i j}$ are rational integers. Since $e_{1}$ is a principal unit, we see that $N(\Omega)$ is a positive integer in $\Omega$. The norm of this positive integer is a nonzero integer in $\Omega$, so that $\Omega$ contains elements of norm not zero.

We may summarize the results of this paragraph in

THEOREM 3. The following five statements are equivalent:

(a) Not every element of the ideal $\Omega$ is of norm 0 .

(b) $\Re$ contains rational integers.

(c) The numbers $\omega_{1}, \omega_{2}, \cdots, \omega_{n}$ of every basis of $\Re$ are linearly independent.

(d) The ideal $\Omega$ is non-singular, i.e., of rank $n$.

(e) The norm $N(\Omega)$ of $\Omega$ is not zero. 
We have shown that each statement implies the next following and that the last implies the first. Thus each of the five statements implies them all.

To see that ideals of norm zero actually exist, we need only to consider the principal ideal $(b]^{*}$ where $N(b)=0$, i.e., where $b$ is a divisor of zero or zero itself. Then $\Delta(b)=0$, and for every number $\lambda$ in $\mathfrak{S}$,

$$
\Delta(\lambda b)=\Delta(\lambda) \cdot \Delta(b)=0
$$

so that $N(\lambda b)=0$ for every number $\lambda b$ in the ideal. Such ideals have none of the properties described in Theorem 3.

4. A condition that $n$ numbers constitute a basis for an ideal. While it is true that every left ideal has a basis $\omega_{1}, \omega_{2}, \cdots, \omega_{n}$ composed of integral numbers, it does not follow that every $n$ numbers of $\subseteq$ constitute a basis for a left ideal. We shall find necessary and sufficient conditions that $n$ numbers $\omega_{1}, \omega_{2}, \cdots, \omega_{n}$ of $\subseteq$ shall form a basis for a left ideal.

Let us assume that $\omega_{1}, \omega_{2}, \cdots, \omega_{n}$ constitute a basis for a left ideal $\Omega$, and that

$$
\omega_{i}=\sum_{j} g_{i j} e_{i} \quad(i=1,2, \cdots, n) .
$$

Every number $k$ of $\Omega$ is of the form

$$
k=\sum_{i} k_{i} \omega_{i}=\sum_{i, j} k_{i} g_{i j} e_{j} .
$$

Every number $s$ of $\subseteq$ is of the form

$$
s=\sum_{l} s_{l} e_{l}
$$

Since $s k$ is in $\Re$, there exist rational integers $d_{r}$ such that

$$
s k=\sum_{l, i, j} s_{l} k_{i} g_{i j} e_{l} e_{j}=\sum_{l, i, j, h} s_{l} k_{i} g_{i j} c_{l j h} e_{h}=\sum_{r, t} d_{r} g_{r t} e_{t} .
$$

Since the basal numbers are linearly independent, this implies

$$
\sum_{l, i, j} s_{i} k_{i} g_{i j} c_{l j t}=\sum_{r} d_{r} g_{r t} \quad(t=1,2, \cdots, n) .
$$

In particular there must exist rational integers $d_{r}$, which we shall call $d_{p q r}$, when $s_{l}=\delta_{l p}$ and $k_{i}=\delta_{i q}$. For these values we have

$$
\sum_{i} g_{q j} c_{p j t}=\sum_{r} d_{p q r} g_{r t} \quad(p, q, t=1,2, \cdots, n) .
$$

* The notation is due to Speiser. See Dickson II, p. 271. 
The existence of integers $d_{p q r}$ satisfying (4.2) is in fact sufficient that the integral numbers $\omega_{1}, \omega_{2}, \cdots, \omega_{n}$ defined by (4.1) constitute a basis for a left ideal. For, let $d_{p q r}$ and $g_{q i}$ be any rational integers satisfying (4.2). Define the $\omega_{i}$ by (4.1). The set of numbers

$$
k=\sum_{i} k_{i} \omega_{i}
$$

where the $k_{i}$ vary independently over $\subseteq$ is evidently closed under addition and subtraction. We can show that it is closed under multiplication on the left by any number $s$ of $\mathfrak{S}$. In fact

$$
s k=\sum_{l, i, j, r} s_{l} k_{i} g_{i j} c_{l j r} e_{r}=\sum_{l, i, r} s_{l} k_{i} \sum_{j} g_{i j} c_{l j r} e_{r} .
$$

Hence by (4.2) we have

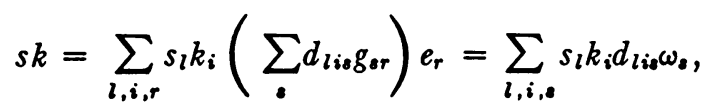

which is obviously of the form $\sum_{i} k_{i}^{\prime} \omega_{i}$.

Let us define the matrices

$C_{p}=\left(c_{p r s}\right)=$ transpose $R_{p}, D_{p}=\left(d_{p r s}\right), G=\left(g_{r s}\right) \quad(p=1,2, \cdots, n)$.

We may now state

Theorem 4.* $A$ necessary and sufficient condition in order that $\omega_{1}, \omega_{2}$, $\cdots, \omega_{n}$ constitute a basis for a left ideal is that there exist integral matrices $D_{1}, D_{2}, \cdots, D_{n}$ such that

$$
G C_{p}=D_{p} G \quad(p=1,2, \cdots, n)
$$

where $G$ is the matrix $\left(g_{\text {res }}\right)$ of (4.1)

5. An explicit basis for a principal ideal. In particular every principal ideal $(d]$ has a basis $\omega_{1}, \omega_{2}, \cdots, \omega_{n}$. We have

$$
d=\sum_{i} d_{i} e_{i}, \quad \omega_{i}=\sum_{i} g_{i j} e_{i} \quad(i=1,2, \cdots, n) .
$$

Let $s=\sum s_{i} e_{i}$ be any number of $\mathfrak{S}$. Then

$$
s d=\sum_{i, j, k} s_{i} d_{j} c_{i j k} e_{k}
$$

is in $(d]$, and hence must be representable in the form

$$
s d=\sum_{i} r_{i} \omega_{i}=\sum_{i, j} r_{i} g_{i j} e_{j} .
$$

- Poincare, using the same correspondence between ideals and matrices, obtained a quite different condition that a matrix in canonical form correspond to an ideal. Bulletin de la Sociéte Mathematique de France, vol. 13 (1885), p. 167. 
It follows that for all rational integers $s_{i}$ there must exist rational integers $r_{i}$ such that

$$
\sum_{i, j} s_{i} d_{j} c_{i j k}=\sum_{i} r_{i} g_{i k} \quad(k=1,2, \cdots, n) .
$$

Conversely, since every linear combination of the $\omega_{i}$ is in $(d]$, it follows that for all integers $r_{i}$ there must exist integers $s_{i}$ such that (5.1) holds. Theșe conditions together are necessary and sufficient in order that $\omega_{1}, \omega_{2}, \cdots, \omega_{n}$ form a basis for the principal left ideal $(d]$.

In particular, let us take $r_{i}=\delta_{h i}$, and denote the corresponding values of $s_{i}$ by $s_{h i}$. Then (5.1) gives

$$
\sum_{i, j} s_{h i} d_{j} c_{i j k}=\sum_{i} \delta_{h i} g_{i k}=g_{h k} \quad(h, k=1,2, \cdots, n) .
$$

Again, let us choose $s_{i}=\delta_{h i}$ in (5.1) and denote the corresponding values of $r_{i}$ by $r_{h i}$. Then we have

$$
\sum_{i, j} \delta_{h i} d_{j} c_{i j k}=\sum_{j} d_{j} c_{h j k}=\sum_{i} r_{h i g} g_{i k} \quad(h, k=1,2, \cdots, n) .
$$

From (5.2) we have, taking matrices,

$$
G=\left(g_{r s}\right)=\left(s_{r s}\right)\left(\sum d_{j} c_{r_{j s}}\right)=\left(s_{r s}\right) S(d)
$$

where $S(d)$ is the transposed second matrix* of $d$. In the same way we obtain from (5.3)

$$
S(d)=\left(r_{r s}\right) G .
$$

Every number of the ideal $(d]$ is of the form

$$
\sum_{i} a_{i} \omega_{i}=\sum_{i, j} a_{i} g_{i j} e_{i}=\sum_{i, k, j} a_{i} s_{i k} \sigma_{k j} e_{i},
$$

where $S(d)=\left(\sigma_{r s}\right)$, and conversely every number of the form $\sum b_{i} \sigma_{i j} e_{i}$ can be written

$$
\sum_{i, j} b_{i} \sigma_{i j} e_{j}=\sum_{i, k, j} b_{i} r_{i k} g_{k j} e_{j}=\sum_{i, k} b_{i} r_{i k} \omega_{k}
$$

and is therefore in $(d]$. Hence the numbers $\sum \sigma_{i j} e_{i}$ constitute a basis for $(d]$.

It follows from the definition of norm that $N((d])=a b s o l u t e$ value $S(d)=$ absolute value $\Delta^{\prime}(d)$. We have now proved

THEOREM 5.1. The principal left ideal (d] has a basis $\omega_{1}, \omega_{2}, \cdots, \omega_{n}$ where

$$
\omega_{i}=\sum_{i} \sigma_{i j} e_{i}, \quad\left(\sigma_{r s}\right)=S(d) ;
$$

the norm of $(d]$ is the absolute value of $\Delta^{\prime}(d)$.

* Dickson I, p. 86, II, p. 35. It is easy to show that $d$ is an integral number if and only if $S(d)$ has integral elements. 
The conditions for associativity may be written

$$
\sum_{j} c_{q k j} c_{p j s}=\sum_{j} c_{p q j} c_{j k s} \text {. }
$$

Let us multiply by $d_{k}$ and sum for $k$ :

$$
\sum_{j, k} d_{k} c_{q k} c_{p j s}=\sum_{j, k} c_{p q j} d_{k} c_{j k s},
$$

which may be written

$$
S(d) C_{p}=C_{p} S(d) \quad(p=1,2, \cdots, n)
$$

where $d=\sum d_{i} e_{i}$. This is exactly (4.2) with $g_{i j}$ replaced by $\sigma_{i j}$ and $d_{p q r}$ replaced by $c_{\text {pqr }}$.

THEOREM 5.2. For every number $d$ in $\subseteq, S(d)$ is commutative with every matrix $C_{p}$; and if $G=S(d)$ in Theorem 4 , then $D_{p}=C_{p}$ for every $p$.

We shall prove later (Theorem 11) that every integral matrix commutative with every $C_{p}$ is the transposed second matrix $S(d)$ of some number $d$ of S.

6. Equivalent ideals. Let $\Omega$ be a non-singular left ideal with basis $\omega_{1}, \omega_{2}, \cdots, \omega_{n}$, and let $s$ be a number of $\subseteq$. If we set

we have

$$
\omega_{i}=\sum_{j} g_{i j} e_{j}, \quad s=\sum_{q} s_{q} e_{q},
$$

$$
\begin{aligned}
\omega_{i} s & \equiv \sum_{j} g_{i j}^{\prime} e_{j}=\sum_{p} g_{i p} e_{p} \sum_{q} s_{q} e_{q} \\
& =\sum_{p, q, r} g_{i p} s_{q} c_{p q q} e_{r} .
\end{aligned}
$$

Hence

$$
g_{i j}^{\prime}=\sum_{p, q} g_{i p} s_{q} c_{p q j}=\sum_{p} g_{i p} \sum_{q} s_{q} c_{p q j}=\sum_{p} g_{i p} \sigma_{p j} .
$$

Taking matrices, we have

But

$$
G^{\prime}=G S(s)
$$

$$
G C_{p}=D_{p} G, \quad S(s) C_{p}=C_{p} S(s),
$$

so that

$$
G^{\prime} C_{p}=G S(s) C_{p}=G C_{p} S(s)=D_{p} G S(s)=D_{p} G^{\prime} .
$$

Therefore $\omega_{1} s, \omega_{2} s, \cdots, \omega_{n} s$ form a basis for an ideal which we may call $\Omega^{\prime}$.

Furthermore, we obtain the same ideal $\Omega^{\prime}$ irrespective of the basis of $\Omega$ with which we start. Using any other basis for $\Omega$, we should have obtained, instead of $G^{\prime}$,

$$
G^{\prime \prime}=A G S(s)
$$


where $A$ is an integral matrix of determinant $\pm 1(\$ 3)$. But $G^{\prime \prime}=A G^{\prime}$ corresponds to the same ideal $\Omega^{\prime}$ as does $G^{\prime}$. For a non-singular ideal $\Omega$ we may call the uniquely existing ideal having the basis $\left(\omega_{1} s, \omega_{2} s, \cdots, \omega_{n} s\right)$ where $\left(\omega_{1}, \omega_{2}, \cdots, \omega_{n}\right)$ is any basis for $\Re$, the ideal $\Re s$.

Two non-singular ideals $\Omega_{1}$ and $\Omega_{2}$ are, according to analogy with the usual definition in algebraic number theory, called equivalent if there exist integral numbers $s_{1}$ and $s_{2}$ of $\subseteq$, neither of norm 0 , such that

$$
\Omega_{1} s_{1}=\Omega_{2} s_{2} .
$$

Let $G_{1}$ and $G_{2}$ be the matrices corresponding to particular bases of $\Omega_{1}$ and $\Omega_{2}$ respectively. The condition for equivalence becomes

$$
G_{1} S\left(s_{1}\right)=A G_{2} S\left(s_{2}\right)
$$

where $A$ is an integral matrix of determinant \pm 1 .

The following theorem is important in showing that the concept of ideal class applies to all semi-simple domains and in pointing to a more comprehensive definition which is applicable to singular ideals as well.

THEOREM 6. A necessary and sufficient condition that two non-singular ideal matrices $G_{1}$ and $G_{2}$ be equivalent is that the corresponding sets of matrices $D_{1 p}, D_{2 p}$ satisfying the equations

$$
G_{1} C_{p}=D_{1 p} G_{1}, \quad G_{2} C_{p}=D_{2 p} G_{2} \quad(p=1,2, \cdots, n)
$$

respectively, be similar-i.e., that $D_{1 p}=A D_{2 p} A^{-1}$ for $p=1,2, \cdots, n$, where $A$ is an integral matrix of determinant \pm 1 .

First, let us suppose that $G_{1}$ and $G_{2}$ are equivalent. We have

$$
\begin{aligned}
G_{1} C_{p} & =D_{1 p} G_{1}, & G_{2} C_{p} & =D_{2 p} G_{2}, \\
G_{1} S\left(s_{1}\right) & =A G_{2} S\left(s_{2}\right), & & |A|= \pm 1
\end{aligned}
$$

where $S\left(s_{1}\right)$ and $S\left(s_{2}\right)$ are each, by Theorem 5.2, commutative with every $C_{p}$. Then

Therefore

$$
\begin{aligned}
G_{1} S\left(s_{1}\right) C_{p} & =G_{1} C_{p} S\left(s_{1}\right)=D_{1 p} G_{1} S\left(s_{1}\right), \\
A G_{2} S\left(s_{2}\right) C_{p} & =A G_{2} C_{p} S\left(s_{2}\right)=A D_{2 p} G_{2} S\left(s_{2}\right) \\
& =A D_{2 p} A^{-1} A G_{2} S\left(s_{2}\right) .
\end{aligned}
$$

$$
D_{1 p} G_{1} S\left(s_{1}\right)=A D_{2 p} A^{-1} G_{1} S\left(s_{1}\right) .
$$

Since both $G_{1}$ and $S\left(s_{1}\right)$ are non-singular, we have

$$
D_{1 p}=A D_{2 p} A^{-1} \quad(p=1,2, \cdots, n) .
$$


Secondly, suppose that the sets of matrices $D_{1 p}$ and $D_{2 p}$ are similar:

$$
G_{1} C_{p}=D_{1 p} G_{1}, \quad G_{2} C_{p}=D_{2 p} G_{2}, \quad D_{1 p}=A D_{2 p} A^{-1}, \quad|A|= \pm 1 .
$$

Then

$$
G_{1} C_{p} G_{1}^{-1}=D_{1 p}=A D_{2 p} A^{-1}=A G_{2} C_{p} G_{2}^{-1} A^{-1} .
$$

We multiply on the left by $G_{2}^{-1} A^{-1}$ and on the right by $G_{1}$, obtaining

$$
G_{2}^{-1} A^{-1} G_{1} C_{p}=C_{p} G_{2}^{-1} A^{-1} G_{1} \text {. }
$$

Let $T$ be the scalar matrix each of whose diagonal elements is $\left|G_{2}\right|$. Then $T G_{2}^{-1} A^{-1} G_{1}$ is an integral matrix and, since it is commutative with each $C_{p}$, it is the second matrix $S(s)$ of some element $s$ of 5 . $^{*}$ Then

$$
T G_{2}^{-1} A^{-1} G_{1}=S(s),
$$

and since $T$ is commutative with every matrix,

$$
G_{1} T=A G_{2} S(s) .
$$

Moreover, $T=S\left(\left|G_{2}\right|\right)$, so that the ideals $\Omega_{1}$ and $\Omega_{2}$ are in fact equivalent.

7. Ideal matrices. An essential point in our proof of Theorem 6 was that $\left|G_{1}\right| \neq 0$. We were therefore unable to consider equivalence of singular ideals. Moreover, the transitive character of equivalence was not apparent from its definition. We now proceed along a line suggested by this theorem but somewhat broader.

We assume a semi-simple rational algebra $\mathfrak{A}$, and a set of integral numbers S of order $n$, the basal numbers being chosen so that the constants $c_{i j k}$ of multiplication are rational integers. As before, we define the matrices $C_{p}=\left(c_{p r s}\right)$ for $p=1,2, \cdots, n$, where $c_{\text {prs }}$ is the element in row $r$ and column $s$. Let $D_{p}=\left(d_{p r a}\right)$ be any set of $n$ integral matrices. All the integral matrices $G$ which satisfy the equations

$$
G C_{p}=D_{p} G \quad(p=1,2, \cdots, n)
$$

will be said to constitute a minor class of ideal matrices, and the set of matrices $D_{1}, D_{2}, \cdots, D_{n}$ will be called a set of corresponding class matrices. The zero matrix at least will satisfy (7.1) no matter how the class matrices may be chosen.

All the matrices of a minor class constitute a modul. In fact, if $G_{1}$, $G_{\mathbf{q}}, \cdots, G_{p}$ are ideal matrices of the same minor class and if $k_{1}, k_{2}, \cdots, k_{p}$ are rational integers, then

$$
G=k_{1} G_{1}+k_{2} G_{2}+\cdots+k_{p} G_{p}
$$

\footnotetext{
* Theorem 11, to follow.
} 
is an ideal matrix of the same minor class. We shall show that if $\mathscr{A}$ is semisimple there exist $r \leqq n$ linearly independent matrices $B_{1}, B_{2}, \cdots, B_{r}$ which constitute a basis for the ideal matrices of the minor class.

LEMMA 7. If $\mathfrak{A}$ is a semi-simple algebra, new basal numbers for $\mathfrak{S}$ and new constants of multiplication $c_{i j k}^{\prime}$ can be so chosen that $\left|c_{r s 1}^{\prime}\right| \neq 0$.

Let us suppose that our basal numbers are chosen as in $\$ 2$, and that the $c_{i j k}$ are defined by

$$
e_{i} e_{j}=\sum_{k} c_{i j k} e_{k} \quad(i, j=1,2, \cdots, n) .
$$

Since $e_{1}$ is a principal unit, $c_{1 j k}=c_{j 1 k}=\delta_{j k}$. If we apply to the basal numbers the transformation

$$
e_{i}=\sum_{j} a_{i j} e_{j}^{\prime}, \quad a=\left|a_{r s}\right| \neq 0,
$$

we get from (7.2):

so that

$$
\begin{aligned}
\sum_{p, q} a_{i p} a_{j q} e_{p}^{\prime} e_{q}^{\prime} & =\sum_{r, \varepsilon} c_{i j r} a_{r \varepsilon} e_{q}^{\prime} \\
& =\sum_{p, q, t} a_{i p} a_{j q} c_{p q}^{\prime} e_{t}^{\prime}
\end{aligned}
$$

$$
\sum_{p, q} a_{r p} a_{s q} c_{p q j}^{\prime}=\sum_{i} c_{r s i} a_{i j} \quad(r, s, j=1,2, \cdots, n),
$$

where the $c_{p q s}^{\prime}$ are defined as in (7.2) with each letter primed. For a fixed $j$, let us form the determinant whose element in row $r$ and column $s$ is (7.4):

$$
a^{2}\left|c_{r r i}^{\prime}\right|=\left|\sum_{i} a_{i j} c_{r s i}\right| \quad(j=1,2, \cdots, n) .
$$

In (7.3) let us now choose

$$
a_{i j}=\tau_{i j} \equiv \sum_{r, k} c_{i j r} c_{r k k} .
$$

If $\mathscr{A}$ is semi-simple, $a=\left|\tau_{r s}\right| \equiv d \neq 0,{ }^{*}$ and

so that

$$
a_{r 1}=\sum_{h, k} c_{r 1 h} c_{h k k}=\sum_{h, k} \delta_{h r} c_{h k k} \equiv \sum_{k} c_{r k k}
$$

$$
\sum_{r} a_{r 1} c_{i j r}=\sum_{r, k} c_{i j r} c_{r k k}=\tau_{i j} .
$$

* Dickson I, p. 108. 
From (7.5) with $j=1$ we obtain

so that $\left|c_{r s 1}^{\prime}\right| \neq 0$.

$$
d^{2}\left|c_{r s 1}^{\prime}\right|=d
$$

In making this transformation we may have lost the property that the basal numbers of $\mathfrak{A}$ form a basis for the set $\subseteq$ of integral numbers. We must now restore this property.,

Bring* the fractions $c_{i j k}^{\prime}$ to a common denominator $\delta$, write $\delta c_{i j k}^{\prime}=h_{i j k}$ where the $h_{i j k}$ are all integers. Set $\epsilon_{i}=\delta e_{i}^{\prime}$. Then

$$
\epsilon_{i} \epsilon_{j}=\delta^{2} e_{i}^{\prime} e_{j}^{\prime}=\delta^{2} \sum_{k} c_{i j k}^{\prime} e_{k}^{\prime}=\sum_{k} h_{i j k} \epsilon_{k} .
$$

We use the new basal numbers $\epsilon_{i}$ whose constants $h_{i j k}$ of multiplication are rational integers. We note that

$$
\left|h_{r s 1}\right|=\left|\delta c_{r s 1}^{\prime}\right|=\delta^{n}\left|c_{r s 1}^{\prime}\right| \neq 0 \text {. }
$$

Proceeding according to the method of Dickson, $\dagger$ we see that every element $x$ of $\mathfrak{S}$ can be put into the form

$$
x=\frac{x_{1}}{D} \epsilon_{1}+\frac{x_{2}}{D} \epsilon_{2}+\cdots+\frac{x_{n}}{D} \epsilon_{n}
$$

where

$$
D=\left|\sum_{i, k} h_{r s i} h_{i k k}\right| \neq 0
$$

and where the $x_{i}$ are rational integers. Of all numbers $x$ in $\mathfrak{S}$ having $x_{1}=x_{2}=\cdots=x_{r-1}=0$ but $x_{r} \neq 0$, choose one having $x_{r}>0$ and minimal for $\epsilon_{r}^{\prime}$. If there is no $x$ of this type having $x_{r} \neq 0$, choose $\epsilon_{r}^{\prime}=0$. We have

$$
\begin{aligned}
& D \epsilon_{1}^{\prime}=b_{11} \epsilon_{1}+b_{12} \epsilon_{2}+\cdots+b_{1 n} \epsilon_{n}, \\
& D \epsilon_{2}^{\prime}=b_{22} \epsilon_{2}+\cdots+b_{2 n} \epsilon_{n}, \\
& D \epsilon_{n}^{\prime}=\quad b_{n n} \epsilon_{n} .
\end{aligned}
$$

Now $\left(\epsilon_{1}^{\prime}, \epsilon_{2}^{\prime}, \cdots, \epsilon_{n}^{\prime}\right)$ form a basis for $\mathfrak{S}$. For, let

$$
x=\frac{1}{D}\left(x_{1} \epsilon_{1}+x_{2} \epsilon_{2}+\cdots+x_{n} \epsilon_{n}\right)
$$

* Dickson I, pp. 161-162.

† Dickson I, p. 162. 
be any number of $\mathfrak{S}$. Set

Then

$$
x_{1}=q_{1} b_{11}+r_{1} \quad\left(0 \leqq r_{1}<b_{11}\right) .
$$

$$
x-q_{1} \epsilon_{1}^{\prime}=\frac{1}{D}\left(r_{1} \epsilon_{1}+x_{2}^{\prime} \epsilon_{2}+\cdots+x_{n}^{\prime} \epsilon_{n}\right)
$$

has its coefficient of $\epsilon_{1}$ less than $b_{11}$ which was minimal, so $r_{1}=0$. Similarly, set

$$
x_{2}^{\prime}=q_{2} b_{22}+r_{2} \quad\left(0 \leqq r_{2}<b_{22}\right) .
$$

Then $x-q_{1} \epsilon_{1}^{\prime}-q_{2} \epsilon_{2}^{\prime}$ lacks the basal numbers $\epsilon_{1}$ and $\epsilon_{2}$. Proceeding in this way, we have after $n$ steps:

$$
x=q_{1} \epsilon_{1}^{\prime}+q_{2} \epsilon_{2}^{\prime}+\cdots+q_{n} \epsilon_{n}^{\prime}
$$

where the $q_{i}$ are rational integers. Since $\mathfrak{S}$ is of order $n$, we now know that $b_{i i} \neq 0$ for every $i$.

Since the numbers $\epsilon_{1}^{\prime}, \epsilon_{2}^{\prime}, \cdots, \epsilon_{n}^{\prime}$ form a basis for $\mathbb{S}$, we know that the new constants $h_{i j k}^{\prime}$ of multiplication are rational integers. Transformation (7.7) can be written

$$
\epsilon_{i}=\frac{D}{b} \sum_{j} B_{j i} \epsilon_{j}^{\prime} \quad(i=1,2, \cdots, n)
$$

where $b=\left|b_{r s}\right|$ and $B_{r s}$, the cofactor of $b_{r s}$, is zero for $r<s$. From (7.5) we have

$$
a^{2}\left|h_{r s 1}^{\prime}\right|=\left|\sum_{i} a_{i 1} h_{r s i}\right|
$$

with $a_{i j}=(1 / b) D B_{j i}=0$ for $i>j$. Hence

$$
a^{2}\left|h_{r s 1}^{\prime}\right|=a_{11}^{n}\left|h_{r s 1}\right| \neq 0
$$

and therefore $\left|h_{r s 1}^{\prime}\right| \neq 0$.

Since the basal numbers $e_{1}, e_{2}, \cdots, e_{n}$ with which we started and the numbers $\epsilon_{1}^{\prime}, \epsilon_{2}^{\prime}, \cdots, \epsilon_{n}^{\prime}$ each form a basis for the set $\subseteq$, we know that there are transformations with rational integral coefficients and of determinants \pm 1 carrying each set of basal numbers into the other set.

8. Effect of change of basis on the fundamental matrices. Let us make a change of basis (7.3) where the $a_{r s}$ are rational integers of determinant \pm 1 . By (7.4) we have

$$
\sum_{r} c_{p i r} a_{r t}=\sum_{s, q} a_{p s} a_{i q} c_{s q t}^{\prime}
$$

Multiply by the cofactor $A_{j t}$ of $a_{j t}$ and sum for $t$. Then 


$$
a c_{p i j}=\sum_{q, t} a_{i q}\left[\sum a_{p s} c_{s q t}^{\prime}\right] A_{j t} .
$$

Passing to matrices, we have

$$
C_{p}=A\left[\sum_{s} a_{p s} C_{s}^{\prime}\right] A^{-1} \quad(p=1,2, \cdots, n)
$$

where $A \equiv\left(a_{r s}\right)$ as our induced transformation on the matrices $C_{p \text {. }}$.

We shall more generally define the matrices $D_{p}^{\prime}$, which will be said to correspond to the matrices $D_{p}$ under transformation (7.3) of determinant \pm 1 , as the solutions of the equations

$$
D_{p}=A\left[\sum a_{p s} D^{\prime}\right] A^{-1} \quad(p=1,2, \cdots, n) .
$$

The matrices $D_{p}^{\prime}$ are evidently integral if the matrices $D_{p}$ are integral. Our definition is justified by the following result:

LEMMA 8.1. If $G$ is an ideal matrix satisfying the equations

$$
G C_{p}=D_{p} G
$$$$
(p=1,2, \cdots, n),
$$

then $G^{\prime}=A^{-1} G A$ satisfies the equations

$$
G^{\prime} C_{p}^{\prime}=D_{p}^{\prime} G^{\prime} \quad(p=1,2, \cdots, n)
$$

where $C_{p}^{\prime}$ and $D_{p}^{\prime}$ are given by (8.1).

From (8.1) and the equations $G C_{p}=D_{p} G$, we have

$$
G A\left[\sum_{\bullet} a_{p s} C_{\bullet}^{\prime}\right] A^{-1}=A\left[\sum a_{p s} D_{\bullet}^{\prime}\right] A^{-1} G,
$$

and therefore

$$
A^{-1} G A\left[\sum_{0} a_{p s} C_{!}^{\prime}\right]=\left[\sum_{\bullet} a_{p s} D^{\prime}\right] A^{-1} G A \text {. }
$$

If we multiply by the cofactor $A_{p r}$ of $a_{p r}$ and sum for $p$, we obtain

$$
A^{-1} G A C_{r}^{\prime}=D_{r}^{\prime} A^{-1} G A \text {, }
$$

which proves the lemma.

LEMMA 8.2. If the basis of $S$ is so chosen that $\left|c_{r o 1}\right| \neq 0$, then an ideal matrix $G$ whose first column consists exclusively of zeros is a zero matrix.

From our definition of ideal matrix we have 
If $g_{k 1}=0$ for every $k$, then

$$
\sum_{k} g_{r k} c_{p k s}=\sum_{k} d_{p r k} g_{k s} \quad(p, r, s=1,2, \cdots, n) .
$$

Taking matrices, we have

$$
\sum_{k} g_{r k} c_{p k 1}=0 \quad(r, p=1,2, \cdots, n)
$$

$$
G\left(c_{s r 1}\right)=0 .
$$

Since the second matrix is non-singular, $G$ must be of rank 0 .

9. A fundamental theorem. Let $\mathfrak{A}$ be a semi-simple algebra, and let $e_{1}, e_{2}, \cdots, e_{n}$ be a basis for a set $\mathfrak{S}$ of integral numbers of $\mathfrak{A}$, where $e_{1}$ is a principal unit. We transform by a matrix $A$ of determinant \pm 1 to another basis $e_{1}^{\prime}, e_{2}^{\prime}, \cdots, e_{n}^{\prime}$ for which $\left|c_{r s 1}^{\prime}\right| \neq 0$. Every ideal matrix $G$ is thereby transformed into an ideal matrix $G^{\prime}=A^{-1} G A$, and inversely $G=A G^{\prime} A^{-1}$, so that there is a one-to-one correspondence between the ideal matrices $G$ and $G^{\prime}$.

If $G^{\prime}$ is an ideal matrix of a certain minor class, so is $-G^{\prime}$. If in the minor class defined by the class matrices $D_{1}^{\prime}, D_{2}^{\prime}, \cdots, D_{n}^{\prime}(\$ 7)$, there are ideal matrices $G^{\prime}=\left(g_{r s}\right)$ in which $g_{11} \neq 0$, define as $B_{1}{ }^{\prime}$ one such matrix in which $g_{11}>0$ and is minimal. If no such matrix exists, set $B_{1}^{\prime}=0$, which surely is in the minor class. If there are in the minor class matrices for which $g_{11}=g_{21}=\cdots=g_{k-1,1}=0$ but $g_{k 1} \neq 0$, define as $B_{k}^{\prime}=\left(b_{k r a}\right)$ one such matrix in which $g_{k 1}=b_{k k 1}>0$ and is minimal, otherwise set $B_{k}^{\prime}=0$. Then $B_{1}^{\prime}$, $B_{2}^{\prime}, \cdots, B_{n}^{\prime}$ form a basis for the matrices of the minor class.

For, let $G^{\prime}=\left(g_{r s}\right)$ be any matrix of the minor class. Set

$$
g_{11}=h_{1} b_{111}+r_{1} \quad\left(0 \leqq r_{1}<b_{111}\right) .
$$

Then $G^{\prime}-h_{1} B_{1}^{\prime}=\left(g_{r}^{\prime}\right)$ is an ideal matrix of the minor class having as its first element $r_{1}$. But $b_{111}$ was minimal, so $r_{1}=0$. Now set

$$
g_{21}^{\prime}=h_{2} b_{221}+r_{2} \quad\left(0 \leqq r_{2}<b_{221}\right) .
$$

Similarly

$$
G^{\prime}-h_{1} B_{1}^{\prime}-h_{2} B_{2}^{\prime}
$$

has two zeros in its first column. Proceeding in this way, we find after $n$ steps that

$$
G^{\prime}-h_{1} B_{1}^{\prime}-h_{2} B_{2}^{\prime}-\cdots-h_{n} B_{n}^{\prime}
$$

is an ideal matrix of the minor class whose first column consists exclusively of zeros, and which by Lemma 8.2 is therefore the zero matrix. Thus

$$
G^{\prime}=h_{1} B_{1}^{\prime}+h_{2} B_{2}^{\prime}+\cdots+h_{n} B_{n}^{\prime}
$$

where $h_{1}, h_{2}, \cdots, h_{n}$ are rational integers. 
Let us now transform back to our original basis. We have $G=A G^{\prime} A^{-1}$, and we define $B_{i} \equiv A B_{i}{ }^{\prime} A^{-1}$, so that every ideal matrix $G$ is expressible in the form

$$
G=h_{1} B_{1}+h_{2} B_{2}+\cdots+h_{n} B_{n}
$$

where the $h_{1}, h_{2}, \cdots, h_{n}$ are rational integers. Conversely, every such matrix is in the minor class. We now have

THEOREM 9. Relative to every basis $e_{1}, e_{2}, \cdots, e_{n}$ for a set $\mathfrak{S}$ of integral elements of a semi-simple rational algebra $\mathfrak{A}$, every minor class of ideal matrices has a basis composed of $n$ matrices $B_{1}, B_{2}, \cdots, B_{n}$ such that the totality of ideal matrices of the minor class is given by

$$
h_{1} B_{1}+h_{2} B_{2}+\cdots+h_{n} B_{n}
$$

where $h_{1}, h_{2}, \cdots, h_{n}$ are independent rational integral variables.

10. Rank of a minor class. Suppose that as in the preceding paragraph we have a set $\subseteq$ of integral numbers with basis so chosen that $\left|c_{\text {rol }}^{\prime}\right| \neq 0$. Then we have seen that every minor class has a basis $B_{1}^{\prime}, B_{2 .}^{\prime}, \cdots, B_{n}{ }^{\prime}$ such that in each matrix $B_{k}{ }^{\prime} \equiv\left(b_{k r s}\right)$ we have $b_{k r 1}=0$ for $r<k$, and either $b_{k k 1}>0$ and minimal, or $B_{k}^{\prime}=0$. Suppose that the $B_{k}^{\prime}$ are linearly dependent:

$$
d_{1} B_{1}^{\prime}+d_{2} B_{2}^{\prime}+\cdots+d_{n} B_{n}^{\prime}=0,
$$

where we may assume that the $d_{i}$ are rational integers not all zero. Considering only elements in the first columns, we have

$$
d_{1} b_{1 r 1}+d_{2} b_{2 r 1}+\cdots+d_{n} b_{n r 1}=0 \quad(r=1,2, \cdots, n) .
$$

Suppose that $d_{1}=d_{2}=\cdots=d_{p-1}=0$ while $d_{p} \neq 0$. Then we have $d_{p} b_{p p 1}=0$ so that $b_{p p 1}=0$ and hence $B_{p}{ }^{\prime}=0$.

If there is a dependence relation among the remaining $n-1$ basal matrices $B_{i}^{\prime}$, we may repeat the argument and show that another one is zero. We finally reach a point where all the basal matrices which are not zero are linearly independent. The number $r$ of linearly independent matrices in a basis is called the rank of the minor class, and if $r<n$ the class is called singular. When we transform to another basis for $\mathfrak{S}$, we see from the relation $B_{i}=A B_{i}^{\prime} A^{-1}$ that the rank is preserved.

Just as in the case of change of basis of an ideal, it can be shown that, relative to the same basis for $\mathfrak{S}$, the most general transformation from one linearly independent basis to another is given by

$$
B_{i}^{\prime}=\sum_{j=1}^{r} a_{i j} B_{i} \quad(i=1,2, \cdots, r)
$$


where $\left(a_{r s}\right)$ is an integral matrix of determinant \pm 1 . The rank is preserved under such transformations also.

11. The principal minor class. All matrices commutative with the fundamental matrices $C_{p}$ evidently constitute a minor class, called the principal minor class. The members of this class we shall call principal ideal matrices. We shall now prove the result required to complete the proof of Theorem 6:

TheOREM 11. Every principal ideal matrix is the transposed second matrix $S(k)$ of some integral number $k$ of $\mathfrak{S}$, and conversely.

The conditions for associativity may be written*

or

$$
\sum_{h} c_{r i h} c_{p h s}=\sum_{h} c_{p r h} c_{h i s}
$$

$$
\left(c_{\text {ris }}\right) C_{p}=C_{p}\left(c_{r i s}\right) \text {. }
$$

That is, the matrices $\left(c_{r i s}\right)=S_{i}=S\left(e_{i}\right)$ belong to the principal minor class. Let $k=k_{1} e_{1}+k_{2} e_{2}+\cdots+k_{n} e_{n}$ be any integral number. Then

$$
S(k)=k_{1} S_{1}+k_{2} S_{2}+\cdots+k_{n} S_{n}
$$

where the $k_{i}$ are rational integers, and therefore $S(k)$ is in the principal minor class. This proves the converse.

We consider now the set of all matrices $G$ which are commutative with every $C_{p}$. We have seen that every such minor class has a basis $B_{1}, B_{2}, \cdots$, $B_{n}$. Since the matrices $S_{i}$ are in this set, we have

$$
S_{i}=\sum_{j} a_{i j} B_{j} \quad(i=1,2, \cdots, n)
$$

where the $a_{i j}$ are rational integers. Now $\left|a_{r s}\right| \neq 0$, since the $S_{i}$ are linearly independent. Therefore we can solve these equations for the $B_{i}$, obtaining

$$
B_{i}=\sum_{j} r_{i j} S_{j}=S\left(r_{i}\right) \quad(i=1,2, \cdots, n)
$$

where each $r_{i j}$ is rational, and $r_{i}=r_{i 1} e_{1}+r_{i 2} e_{2}+\cdots+r_{i n} e_{n}$ is a number of the algebra $\mathfrak{A}$. But $S\left(r_{i}\right)=B_{i}$ is an integral matrix, and hence by the footnote to $\$ 5$ each $r_{i}$ is an integral number and therefore the $r_{i j}$ are rational integers. Then every matrix

$$
G=h_{1} B_{1}+h_{2} B_{2}+\cdots+h_{n} B_{n}
$$

can be written

$$
G=S(k), \quad k=\sum_{i, j} h_{i} r_{i j} e_{j}
$$

* Dickson I, p. 92. 
where the $h_{i}$ and $r_{i j}$ are rational integers, so that $k$ is a number of $\mathfrak{S}$. This completes the proof of the theorem.

12. The class number. We shall now establish a few properties of minor classes.

THEOREM 12.1. If $G$ is an ideal matrix of the minor class $\mathfrak{l}$, and if $P$ is a principal ideal matrix, then GP is an ideal matrix of the minor class $\mathfrak{l}$.

Let $D_{1}, D_{2}, \cdots, D_{n}$ be the class matrices defining the minor class $\mathfrak{l}$. Then

$$
G C_{p}=D_{p} G, \quad P C_{p}=C_{p} P \quad(p=1,2, \cdots, n) .
$$

Therefore

$$
G P C_{p}=G C_{p} P=D_{p} G P,
$$

which proves the theorem.

THEOREM 12.2. If the minor class $\mathfrak{l}$ contains one non-singular ideal matrix $G$, then the $n$ basal matrices of $\mathfrak{l}$ are linearly independent, and $\mathfrak{l}$ is of rank $n$.

Let $G$ be a non-singular matrix of $\mathfrak{l}$. Let $P_{1}, P_{2}, \cdots, P_{n}$ be linearly independent matrices of the principal class. Then $G P_{1}, G P_{2}, \cdots, G P_{n}$ are linearly independent matrices of class $\mathfrak{l}$, for if there were a dependence relation

$$
\sum_{i} d_{i} G P_{i}=G \sum_{i} d_{i} P_{i}=0
$$

where $G$ is a non-singular matrix, we should have $\sum d_{i} P_{i}=0$, contrary to assumption. Let $B_{1}, B_{2}, \cdots, B_{n}$ be a basis for $\mathfrak{l}$. Then

$$
G P_{i}=\sum_{j} b_{i j} B_{j} \quad(i=1,2, \cdots, n),
$$

and since the $G P_{i}$ are linearly independent, so are the basal matrices $B_{j}$. Thus the rank of $\mathfrak{l}$ is $n$.

THEOREM 12.3. If $\mathfrak{A}$ is a division algebra, every minor class except the zero class is non-singular.

Since $\sum d_{i} P_{i}$ is the transposed second matrix of a number of $\mathfrak{A}$, it is either of rank $n$ or of rank 0 . Thus in (12.1) either $G=0$, or else $d_{1}=d_{2}=\cdots$ $=d_{n}=0$ and the matrices $G P_{i}$ are linearly independent.

THEOREM 12.4. If two minor classes contain the same non-singular ideal matrix, the classes coincide. 
Suppose that

$$
G C_{p}=D_{1 p} G, G C_{p}=D_{2 p} G, \quad|G| \neq 0 \quad(p=1,2, \cdots, n) .
$$

Then $D_{1 p} G=D_{2 p} G$, and since $G$ is non-singular, $D_{1 p}=D_{2 p}$ for every $p$. Thus the minor classes coincide.

The theorem is not true with the omission of the word "non-singular." Thus the zero matrix is common to every minor class.

In general we shall not expect the number of minor classes to be finite. Thus $D_{1}, D_{2}, \cdots ; D_{n}$ may be taken as perfectly arbitrary integral matrices, and the equations $G C_{p}=D_{p} G$ will be satisfied by the zero matrix at least. Moreover, if there be matrices satisfying a relation $G C_{p}=D_{p} G$, then

$$
A G C_{p}=A D_{p} A^{-1} A G,
$$

so that the matrices $A D_{p} A^{-1}$ define a minor class in general distinct from the given class.

Two minor classes whose class matrices are connected by a relation

$$
D_{p}^{\prime}=A D_{p} A^{-1},|A|= \pm 1 \quad(p=1,2, \cdots, n)
$$

will be called similar minor classes. We now define the (left) class number $h$ of the set $\mathfrak{S}$ of integral numbers of $\mathfrak{A}$ as the (cardinal) number of dissimilar non-singular minor classes of (left) ideal matrices.

It is evident from Theorem 6 that when $\mathfrak{A}$ is an algebraic field, $h$ becomes the ordinary class number of the field. All ideal matrices corresponding to principal ideals belong to minor classes which are similar to the principal minor class. We have therefore, without using the concept of ideal multiplication, succeeded in generalizing to sets $\subseteq$ of semi-simple algebras the concept of ideal class in a satisfactory manner. For instance, we may prove in the usual manner

TheOREM 12.5. A necessary and sufficient condition in order that every pair of numbers of $\subseteq$ may possess a greatest common right divisor expressible linearly in terms of the numbers is that the left class number $h$ of $\subseteq$ be 1.

13. The density of ideal matrices. It is recognized that the addition of Dedekind ideals cannot be defined in any useful way, because of the fact that associated numbers correspond to the same principal ideal. This is not true of ideal matrices, however, and we have a satisfactory additive theory within each minor class.

Let $B_{1}, B_{2}, \cdots, B_{n}$ be a set of basal matrices for a non-singular minor class 1 . Every ideal matrix of $\mathfrak{l}$ has the form

$$
G=a_{1} B_{1}+a_{2} B_{2}+\cdots+a_{n} B_{n}
$$


where the $a$ 's are rational integers, and conversely every such $G$ is in $\mathfrak{t}$. Since the $B_{i}$ are linearly independent, this representation is unique. Addition and scalar multiplication within the minor class $\mathfrak{t}$ are defined as in the theory of linear algebras, and follow the usual laws.

We have seen that $S_{1}, S_{2}, \cdots, S_{n}$ constitute a basis for the principal minor class ( $\$ 11)$. The numbers

$$
a=a_{1} e_{1}+a_{2} e_{2}+\cdots+a_{n} e_{n}
$$

are in one-to-one correspondence with the principal ideal matrices

$$
S(a)=a_{1} S_{1}+a_{2} S_{2}+\cdots+a_{n} S_{n},
$$

which in turn are in one-to-one correspondence with the ideal matrices $G$ of each class $\mathfrak{l}$, and this correspondence is preserved under addition and scalar multiplication. Thus we have

THEOREM 13. The ideal matrices of every non-singular minor class $\mathfrak{t}$ are in one-to-one correspondence with the numbers of the set $\mathfrak{S}$. This correspondence is preserved under addition and scalar multiplication.

Ohio State University,

Columbus, Orio 